\title{
Designing and Binding Mode Prediction of Juvenile Hormone Analogues as Potential Inhibitor for Galleria mellonella
}

Pamita Awasthi and Priyanka Sharma*

Department of Chemistry, National Institute of Technology, Hamirpur, Himachal Pradesh, India

\begin{abstract}
Virtual screening of chemical databases has become an integral part of ligand design. Docking is one of the most important methods in computer assisted screening. If a three dimensional structure of target receptor is available, along with information regarding nature of the ligand-binding site, ligand-binding mode; the interactions between the ligand and receptor can be studied extensively, in order to design and develop target specific new compounds in a short time period. Juvenile Hormone Analogues, sesqui-terpenoid series of compound act as an insect growth regulator, and presently in use as a potential environment friendly pesticide. Juvenile hormone is the molting hormone responsible for each molt, and involved in a wide range of physiological processes in both developing and mature insect. Designing of various juvenile hormone analogues are new and emerging area to counter the insect problem.

In this paper, we report protein-ligand interactions using a standard protocol of docking. We perform screening of synthesized (A-B) and proposed (C-D) series of Juvenile Hormone Analogues with hemolymph binding proteins of Galleria mellonella. Further binding energy profile of all the series have been compared with the phenoxy derivatives of juvenile hormone mimics, as well as natural $\mathrm{JH}$ III, in order to design targeted JHAs with improved biological activities. Our proposed series of juvenile hormone analogues exhibit better energy profile over in use phenoxy derivatives.
\end{abstract}

Keywords: Virtual screening; Juvenile hormone analogues; Insect growth regulators; Juvenile hormone binding proteins; Docking; Galleria mellonella

\section{Background}

Insects have a unique growth pattern involving different transition stages in their life cycle. These stages are under the control of specific ratio of juvenile hormone. Juvenile hormone $(\mathrm{JH})$ is secreted by corpora allata of insect, and carried by different proteins to target site. The level of JHs in hemolymph fluctuates during the development of an insect's stadium and correlates with specific developmental events, such as molting and metamorphosis. A slight variation in the ratio of this hormone leads to physiological deformities in insect species. This leads to vary the growth pattern in insects, which ultimately affect their population.

A number of new methods and tools have been proposed in the direction to develop such compounds capable to interfere with the processes of growth, development and metamorphosis, i.e. life cycle of the target insect. These compounds have been called as an insect growth regulator (IGR), or third generation insecticides. They have been classified mainly in two categories that mimic the action of insect growth and developmental hormone, the ecdysteroids and the juvenile hormones.

Juvenile hormone binding protein (JHBP) transports juvenile hormone $(\mathrm{JH})$ from corpora allata to target cells, and serves as a pool of $\mathrm{JH}$ in the hemolymph. $\mathrm{JH}$ regulates growth, development and metamorphosis of insects. Its presence maintains larval stage, and absence allows metamorphosis of insect to occur. In hemolymph, three separate groups of proteins may bind $\mathrm{JH}$, lipophorins, hexameric proteins and low molecular weight proteins. Complex of JHBP-JH gets stronger with the presence of other lipid proteins present in the hemolymph. The Area of our study is Greater wax moth. Among the wax moth pests of the honeybee, the greater wax moth Galleria mellonella L. (Lepidoptera: Pyralidae) causes the greatest damage, leading to material and financial losses.
The larval stage of G. mellonella builds silk-lined feeding tunnel in the honeycomb and feeds on wax, pollen, faeces and cocoon of the bee larvae. This voracious nature of the larva leads to the destruction of the honeycomb, and subsequent to the death of weak colonies. Adults do not feed, because they have atrophied mouth parts. The greater wax moth can be controlled via biological, physical, and chemical methods, but most of these methods are either inefficient or expensive for the small-scale beekeeper. The structure of JHBP, along with binding pocket of G. mellonella has been solved, which ultimately provides the base for computer aided designing. JHBP from G. mellonella is a glycoprotein having 226 amino acid residues. Structurally, it resembles the folding pattern of some mammalian lipid-binding proteins. The JHBP molecule undergoes a profound conformational transition upon binding $\mathrm{JH}$ [1-5]. This provides a base for rational designing of different analogues of $\mathrm{JH}$. These synthetic derivatives cause stimulation or inhibition of $\mathrm{JH}$ titer, and ultimately disturb the physiology of an insect. Among these, need is to develop species-specific environmentfriendly pesticides.

Therefore, knowledge of target site of juvenile hormone receptor $(\mathrm{JH})$ is attaining a lot of importance for the development of novel insecticides. Molecular modeling allows evolution of several alternatives before experimental works are carried out. Molecular docking is one of the widely used modeling tools for the study of molecular recognition,

${ }^{*}$ Corresponding author: Priyanka Sharma, Department of Chemistry, National Institute of Technology, Hamirpur, Himachal Pradesh-17-7005, India, Tel: +91 01972-254140; Fax: +91-01972-2223834; E-mail: priyankasharma@nith.ac.in

Received May 13, 2013; Accepted June 17, 2013; Published June 20, 2013

Citation: Pamita A, Priyanka S (2013) Designing and Binding Mode Prediction of Juvenile Hormone Analogues as Potential Inhibitor for Galleria mellonella. J Comput Sci Syst Biol 6: 106-111. doi:10.4172/jcsb.1000107

Copyright: ( 2013 Pamita A, et al. This is an open-access article distributed unde the terms of the Creative Commons Attribution License,which permits unrestricted use, distribution, and reproduction in any medium, provided the original author and source are credited. 
which aims to predict the binding mode and binding affinity of proteinligand complex.

In this paper, molecular modeling tool is employed for four different series (A, B, C and D) of JHAs, along with natural JH III and phenoxy derivatives with JHBP of G. mellonella, to understand the mode of interactions. A and B series of JHAs were synthesized earlier and studied for their biological activity [6,7]. Based upon structure activity relationship, we propose two different series $\mathrm{C}$ and $\mathrm{D}$ as an analogue of JH. Designing of these analogues have been done in a way to have maximum interactions within the binding pocket. Higher the interactions; stronger will be the complex of amino acid residues lining of the pocket and the ligand. Various structural modifications have been incorporated to the parent molecule, in order to enhance the activity within the binding cavity. Here, we report the comparative binding free energy analysis of all the analogues of four different series. Further we compare our findings with natural JH III and phenoxy derivatives. Finally designed ligands are ranked according to the binding affinities with the target protein.

\section{Methodology}

The structures of ligands were built using pymol software tool (www.pymol.com), and optimized with AMBER force field of the AutoDock 4.2. AutoDock tools (ADT) (version 1.5.4) were used for protein and ligand preparation. Briefly for protein, all hydrogen, including non-polar, Kollman charges and solvation parameters were added to all atoms. After adding charges, the non-polar hydrogens were merged. Same process followed for the ligand molecules. Autogrid was used to generate the grid maps. The docking area was defined using AutoGrid $60 \times 60 \times 60-3 \mathrm{D}$ affinity grid centered on the binding site of receptor protein, with a $0.375 \AA$ Arid point space was identified. For all ligands, Gasteiger charges were assigned, and then nonpolar hydrogen was merged. Lamarckian genetic algorithm (LGA) was employed for ligand conformational searching, because it has enhanced performance relative to simulated annealing or the simple genetic algorithm [8]. For each compound, we used the default docking parameters, with the exception of the followings: initial population of 150 randomly placed individuals, maximum number of $2.5 \times 10^{6}$ energy evaluations and maximum number of $2.7 \times 10^{4}$ generations. The mutation rate and crossover rate were set to 0.02 and 0.80 , respectively. The elitism value was set to 1 , and the local search frequency to 0.06 . Hundred independent docking runs were carried out for each ligand, using these parameters for rapid screening. The best docked position was determined by comparing docking poses and considering the total energy value.

Among several similar docking poses, the more energetically favorable conformation was selected. The docking results were clustered on the basis of root-mean-square deviation between the Cartesian coordinates of the atoms using $2.0 \AA$ A cutoff, and were ranked on the basis of the binding free energy $\left(\Delta \mathrm{G}_{\mathrm{b}}, \mathrm{kcal} / \mathrm{mol}\right)$ and inhibitory constant $(\mathrm{Ki}, \mu \mathrm{M})$.

(All the calculations of Autogrid and Autodock are performed on Linux operating system with system Properties (Intel(R) Pentium(R) D CPU $2.80 \mathrm{GHz}, 4.0 \mathrm{~GB}$ of RAM).

\section{Results and Discussion}

Docking methods have been very commonly used to suggest the binding modes of ligands with proteins $[9,10]$. Autodock 4.2 software module has been used to identify the potential binder in A to D series.
LGA protocol has been applied with set parameter, in order to check the docking performance of designed ligands in comparison to synthesized ones. All ligands from A to D series are compared based upon their binding free energy (B.E.) profile. Formula used for calculating binding energy.

$$
\Delta \mathrm{G}_{\text {binding }}=\Delta \mathrm{G}_{\text {vdw }}+\Delta \mathrm{G}_{\text {ele }}+\Delta \mathrm{G}_{\text {desol }}+\Delta \mathrm{G}_{\text {int }}+\Delta \mathrm{G}_{\text {tor }}+\Delta \mathrm{G}_{\text {unbound }}
$$

Inhibitory constant (Ki) is also determined and compared. All the analogues interacted in same fashion, and showing hydrogen bonding with amino acid residues at binding cavity. Correct binding/geometry of ligand inside the protein cavity is not only important for molecular recognition, but also in lead identification. Specific hydrogen bonding and hydrophobic interaction between atoms of ligand and protein leads to dramatic conformational change of JHBP $[2,3]$. This conformational change is a signal for physiological changes in insect life cycle. Any changes like secretion decay/early, etc. is responsible for physiological deformation in insects.

Computational applications are essential for rational design of new analogues. Models that are capable to explain the interactions and predict the biological activity of compounds by their structural properties have been considered as powerful tools to design highly active molecules. In molecular docking, we attempt to predict the structure (or structures) of the intermolecular complex formed between two or more molecules [11,12]. Phenoxy derivative of JHAs found to be more bio effective, in comparison to the terpenoid JHAs [13]. Based upon this information emphasis has been given to develop phenoxy, rather than terpenoid skeleton for $\mathrm{JH}$ analogues. Proposed $\mathrm{C}$ and $\mathrm{D}$ series bears different functionality at the main chain, aromatic/heterocyclic rings at the terminal position. Variations have been done at the terminal group $\left(\mathrm{R}_{1}\right)$ of the parent compound by incorporating different functionalities like aniline, substituted aniline, phenol, substituted phenols, five/six member heterocyclic rings like pyrrolidine, piperidine and morpholine. In addition to this functionality, the hydrophobicity of the compounds has been varied by incorporating different structural features at the main chain (C and D) of the series. Variations also have been done at $\mathrm{R}$ group at ring $\mathrm{Y}$ (Figure 1). Amino acids which are<smiles>[R]C(=O)C([Y])NS(=O)(=O)c1ccc([R])cc1</smiles>
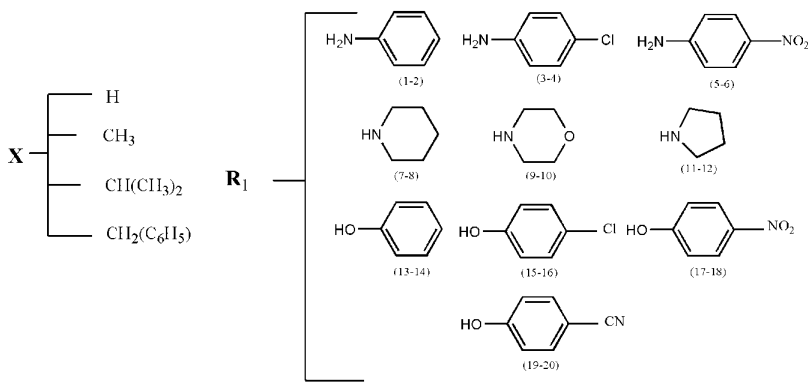

$\mathbf{R}=\mathrm{H}, \mathrm{CH}_{3}$

Compound 1,3,5,7, $11,13,15,17,19: \mathbf{R}=\mathbf{H}$ Compound 2,4,6,8,10,12,14,16,18,20: $\mathbf{R}=\mathbf{C H}_{3}$

Figure 1: Structure of parent compound of synthesized and proposed series (A-D). 
Citation: Pamita A, Priyanka S (2013) Designing and Binding Mode Prediction of Juvenile Hormone Analogues as Potential Inhibitor for Galleria mellonella. J Comput Sci Syst Biol 6: 106-111. doi:10.4172/jcsb.1000107

non polar in nature have been incorporated at point $\mathrm{X}$ to develop the hydrophobic environment around the molecule, to strengthen JHBPJHAs complex inside the binding cavity. Therefore, hydrophobicity of the analogues has been increased, keeping in mind the size, as well as volume of the binding pocket of receptor protein.

Sulfonamide functionality has been added at the main chain of the molecule. Sulfonamide group exhibit various properties like fungicides, insecticidal, anti bacterial, anti cancerous, etc. [14,15]. Keeping in view the multifunction behavior of sulfonamide, this group has effectively added at the main chain of JHAs. Further, in order to provide more flexibility to ligand skeleton, various labile groups have been added at the main chain, which provides flexibility as well as availability of more interaction sites to the amino acid residues of the binding cavity.

Overall, designing of analogues have been done by developing phenoxy skeleton, oxo, oxa as well as aza linkage, in order to occupy the binding cavity effectively. As hydrogen bonding and hydrophobic interactions are prerequisite for mediating behavior of receptor protein during JHBP-JH complex, so we tried to increase hydrogen bonding potential of ligands by incorporating the above said functional groups at the main chain.

All these structural modifications in the ligand molecule need proper validation. In order to support this theoretical model, computational designing has been chosen prior to synthesis for the development of potent compounds. Docking analysis of designed analogues with JHBP of G. mellonella has been carried out in depth. We studied the receptor-ligand interactions, as well as their binding mode for all the analogues of A-D series, with JHBP of G. mellonella. Interactions of the proposed series $\mathrm{C}$ and $\mathrm{D}$ have been compared with the previous synthesized series $\mathrm{A}$ and $\mathrm{B}$, natural $\mathrm{JH}$ III and synthetic IGRs like fenoxycarb, S-21149, Compound 1 and Pyriproxyfen.

It is clear from the Figure 2; there occur the sharp lowering in binding energy profile of all the analogues. Comparing all the series (AD); C and D series show the greater lowering in binding energy profile, as compared to previously synthesized A and B series and synthetic IGRs. Among C and D series, analogues of D series further exhibit the greater lowering in binding energy profile.

The total binding free energy, $\Delta \mathrm{G}_{\mathrm{b}}$, for all protein-ligand complexes are summarized and further compared with natural JH III and synthetic IGRs (Table 1a and 1b). To further understand major determinants of binding, each is comprised of various contributions, including vander Waals energy, electrostatic energy, solvation free energy, internal, torsional energy and entropy. Both vanderwaal and electrostatic terms mainly contributes towards binding of ligand with protein, and play key role in the bioactivities of these compounds. Hydrogen bonding is the additive effect of Vanderwaal and electrostatic interactions. Binding free energy behavior of designed analogues is highly different in comparison to natural JH III and synthetic IGRs.

Analogues 1-6 for all the series (A-D) show a uniform trend for lower binding energy profile. Analogues of the proposed series $(C$ and D), exhibit greater lowering of $\Delta \mathrm{G}_{\text {bind }}$. This could be due to the effective binding mechanism of these analogues inside the pocket of the receptor protein. Earlier synthesized bioactive series (A and B) comparatively exhibit higher binding energy profile, this could be due to the improper interaction of these analogues inside the binding pocket. Further various structural modifications of proposed $\mathrm{C}$ and $\mathrm{D}$ series analogues leads the better fit, as well as better interactions inside the binding pocket of the receptor protein. $5^{\text {th }}$ analogue of $\mathrm{D}$ series shows H-bonding interactions with LYS 85 and ALA 220 amino acid residue inside the pocket, in comparison to the same analogue of the other series. Likewise, $6^{\text {th }}$ analogue of $\mathrm{A}$ and $\mathrm{B}$ series shows the effective interaction with LYS 218 amino acid inside the binding pocket. Both $\mathrm{NO}_{2}$ as well as $-\mathrm{NH}$ functionalities are participating effectively inside the pocket. In comparison to this analogue of series

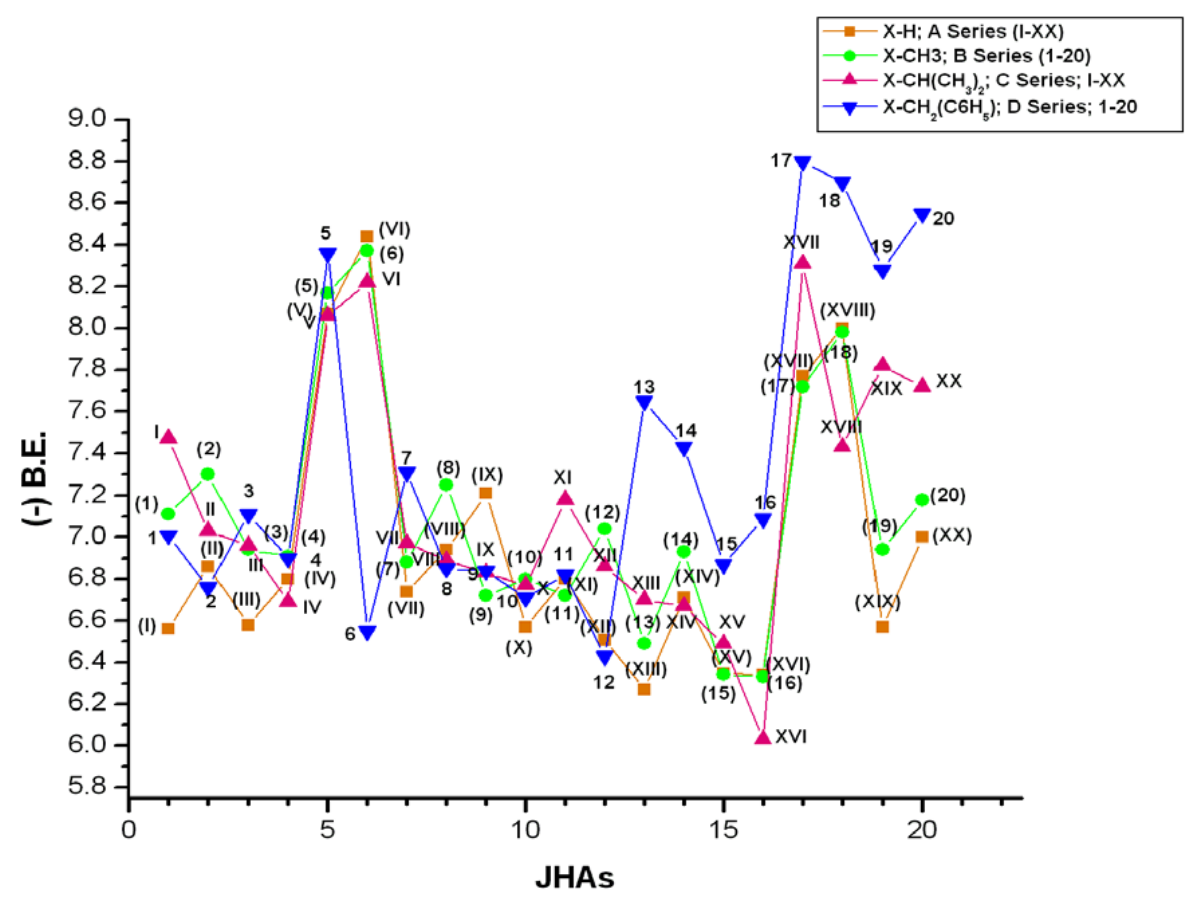

Figure 2: Binding energy profile $\left(\Delta G_{b}\right.$ in $\left.\mathrm{Kcal} / \mathrm{mol}\right)$ of synthesized $(A-B)$ and proposed $(C-D)$ series analogues 
Citation: Pamita A, Priyanka S (2013) Designing and Binding Mode Prediction of Juvenile Hormone Analogues as Potential Inhibitor for Galleria mellonella. J Comput Sci Syst Biol 6: 106-111. doi:10.4172/jcsb.1000107

\begin{tabular}{|c|c|c|c|c|c|c|c|c|c|}
\hline \multirow{2}{*}{ S.No. } & \multirow[b]{2}{*}{ IDs } & \multicolumn{2}{|c|}{$\begin{array}{l}\text { (A) } \\
X-H\end{array}$} & \multicolumn{2}{|c|}{$\begin{array}{c}(\mathrm{B}) \\
\mathrm{X}-\mathrm{CH}_{3}\end{array}$} & \multicolumn{2}{|c|}{$\begin{array}{c}(\mathrm{C}) \\
\mathrm{X}-\mathrm{CH}\left(\mathrm{CH}_{3}\right)_{2}\end{array}$} & \multicolumn{2}{|c|}{$\begin{array}{c}(\mathrm{D}) \\
\mathrm{X}-\mathrm{CH}_{2}\left(\mathrm{C}_{6} \mathrm{H}_{5}\right)\end{array}$} \\
\hline & & $\begin{array}{c}\text { B.E. } \\
\text { (Kcal/mol) }\end{array}$ & $\begin{array}{c}\mathbf{K i} \\
\left(\mu \mathrm{M}, \mathrm{nM}^{*}\right)\end{array}$ & $\begin{array}{c}\text { B.E. } \\
\text { (Kcal/mol) }\end{array}$ & $\begin{array}{c}\mathbf{K i} \\
\left(\mu \mathrm{M}, \mathrm{nM}^{*}\right)\end{array}$ & $\begin{array}{c}\text { B.E. } \\
\text { (Kcal/mol) }\end{array}$ & $\begin{array}{c}\mathbf{K i} \\
\left(\mu \mathrm{M}, \mathrm{nM} \mathbf{M}^{*}\right)\end{array}$ & $\begin{array}{c}\text { B.E. } \\
\text { (Kcal/mol) }\end{array}$ & $\begin{array}{c}\mathbf{K i} \\
\left(\mu \mathrm{M}, \mathrm{nM}^{*}\right)\end{array}$ \\
\hline I. & 1 & -6.56 & 15.50 & -7.11 & 6.09 & -7.47 & 3.35 & -7.01 & 7.24 \\
\hline II. & 2 & -6.86 & 9.39 & -7.3 & 4.48 & -7.03 & 7.03 & -6.76 & 11.18 \\
\hline III. & 3 & -6.58 & 14.98 & -6.94 & 8.15 & -6.96 & 7.98 & -7.11 & 6.14 \\
\hline IV. & 4 & -6.8 & 10.31 & -6.91 & 8.66 & -6.69 & 12.49 & -6.9 & 8.74 \\
\hline V. & 5 & -8.07 & 1.22 & -8.17 & 1.03 & -8.06 & 1.23 & -8.36 & $744.16^{*}$ \\
\hline VI. & 6 & -8.44 & $645.48^{*}$ & -8.37 & $734.56^{*}$ & -8.22 & $941.82^{\star}$ & -6.55 & 15.87 \\
\hline VII. & 7 & -6.74 & 11.37 & -6.88 & 8.98 & -6.97 & 7.74 & -7.31 & 4.37 \\
\hline VIII. & 8 & -6.94 & 8.13 & -7.25 & 4.87 & -6.89 & 8.85 & -6.85 & 9.51 \\
\hline IX. & 9 & -7.21 & 5.18 & -6.72 & 11.86 & -6.83 & 9.91 & -6.84 & 9.63 \\
\hline$x$ & 10 & -6.57 & 15.35 & -6.8 & 10.43 & -6.77 & 10.96 & -6.71 & 1.08 \\
\hline XI. & 11 & -6.8 & 10.39 & -6.72 & 11.78 & -7.18 & 5.47 & -6.82 & 10.01 \\
\hline XII. & 12 & -6.51 & 16.86 & -7.04 & 6.87 & -6.86 & 9.36 & -6.43 & 19.27 \\
\hline XIII. & 13 & -6.27 & 25.15 & -6.49 & 17.38 & -6.7 & 12.20 & -7.65 & 2.47 \\
\hline XIV. & 14 & -6.71 & 11.98 & -6.93 & 8.39 & -6.67 & 12.87 & -7.43 & 3.60 \\
\hline $\mathrm{XV}$ & 15 & -6.35 & 22.11 & -6.34 & 22.51 & -6.49 & 17.51 & -6.87 & 9.17 \\
\hline XVI. & 16 & -6.34 & 22.46 & -6.33 & 22.80 & -6.03 & 37.83 & -7.09 & 6.33 \\
\hline XVII. & 17 & -7.77 & 2.02 & -7.72 & 2.20 & -8.31 & $811.99^{*}$ & -8.8 & $357.30^{*}$ \\
\hline XVIII. & 18 & -8 & 1.37 & -7.98 & 1.41 & -7.43 & 3.57 & -8.7 & $420.74^{*}$ \\
\hline XIX. & 19 & -6.57 & 15.41 & -6.94 & 8.13 & -7.82 & 1.84 & -8.28 & $846.23^{*}$ \\
\hline$X X$. & 20 & -7 & 7.40 & -7.18 & 5.41 & -7.72 & 2.21 & -8.55 & $541.15^{*}$ \\
\hline
\end{tabular}

Table 1a: Free energy of binding $\left(\Delta \mathrm{G}_{\mathrm{b}}, \mathrm{Kcal} / \mathrm{mol}\right)$ of synthesized (A and B) and proposed (C and D) series of Juvenile Hormone Analogues with JHBP of G. mellonella.

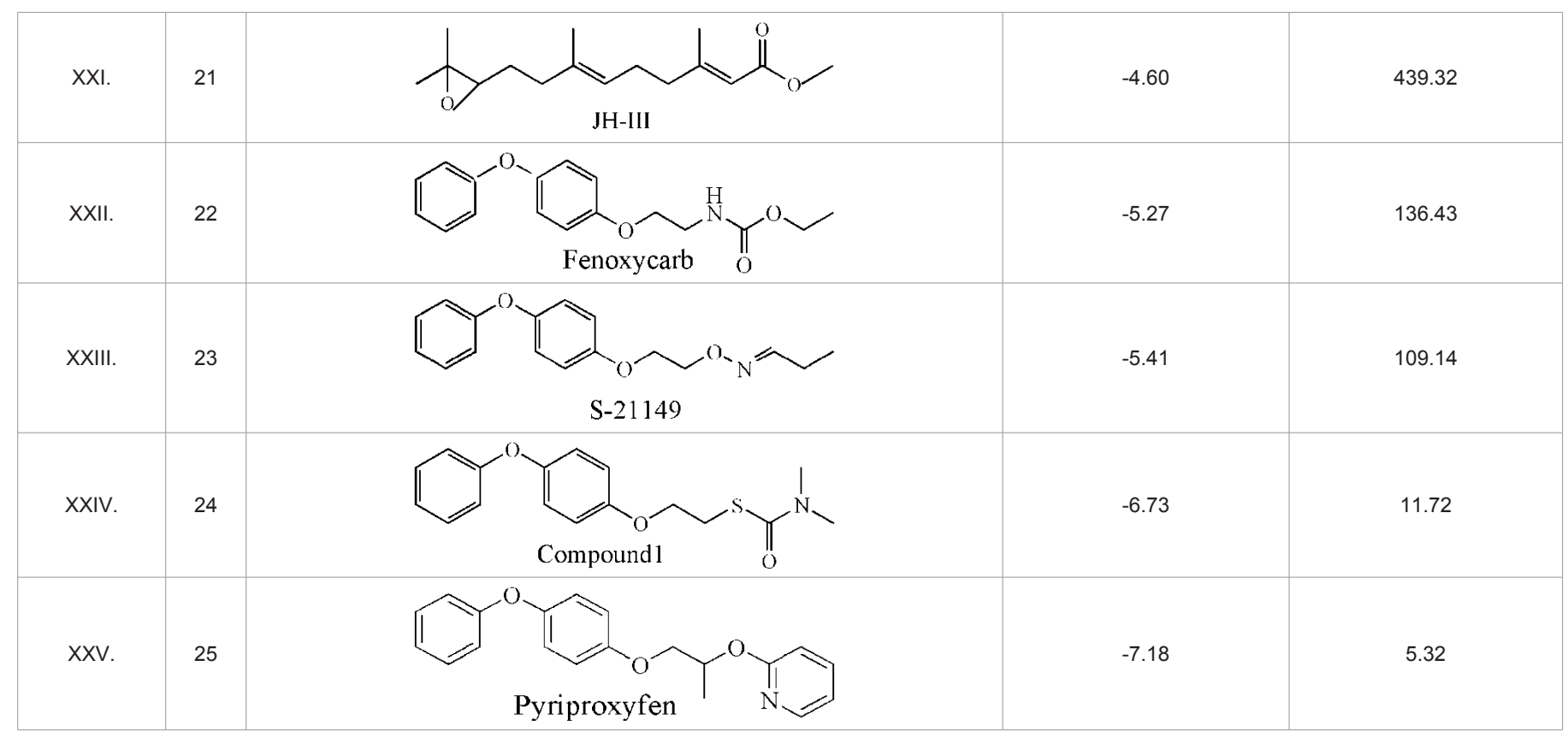

Table 1b: Free energy of binding $\left(\Delta \mathrm{G}_{\mathrm{b}}, \mathrm{Kcal} / \mathrm{mol}\right)$ of natural JH III, Fenoxycarb, S-21149, Compound 1 and Pyriproxyfen (IGRs) with JHBP of G. mellonella. 
Citation: Pamita A, Priyanka S (2013) Designing and Binding Mode Prediction of Juvenile Hormone Analogues as Potential Inhibitor for Galleria mellonella. J Comput Sci Syst Biol 6: 106-111. doi:10.4172/jcsb.1000107

$\mathrm{A}$ and $\mathrm{B}$, same analogue of $\mathrm{C}$ series exhibit better binding energy behavior. This could be due to the effective arrangement of the various functionalities present at the main chain skeleton of the analogue 6 . Analogue 6 shows $\mathrm{H}$-bonding interactions with TYR 130, with -NH and $\mathrm{NO}_{2}$ with LYS 85. Similarly, Analogue 17 of $\mathrm{C}$ and $\mathrm{D}$ series shows $\mathrm{H}$-bonding interactions with LYS 85. In addition to this, analogue of D series shows additional H-bonding interaction with LYS 218. It is the structural arrangement of the analogues inside the binding pocket of receptor protein, which determines the binding mode. 18, 19 and $20^{\text {th }}$ analogues of $\mathrm{D}$ series shows $\mathrm{H}$-bonding interactions with LYS 85 and LYS 218 amino acid residues of the pocket. Analogues of D series exhibits $\pi$-cation interactions, in addition to $\mathrm{H}$-bonding, due to the presence of aromatic ring at the main chain at point $\mathrm{X}$. The presence of this aromatic ring further stabilizes the JHBP-JHAs complex inside the binding cavity (Figure 3).

However, analogues from 7-12 of all the series (A-D) bears the elevation in binding energy profile, in comparison to other analogues. This may be due to the presence of heterocyclic rings at terminal position $\mathrm{R}_{1}$. Among these heterocyclic, there is lack of resonance effect. There is no delocalization of charges in these rings, which lowers the availability of the free attacking site at terminal end $\mathrm{R}_{1}$. Overall cyclic rings do not participate effectively for the binding inside the pocket of receptor protein. It is the overall structure of the compound, which determines the effectiveness of the compound. Increase in hydrophobicity may cause the steric hindrance with cyclic rings at position $\mathrm{R}_{1}$, which could be the reason of increase in binding free energy.
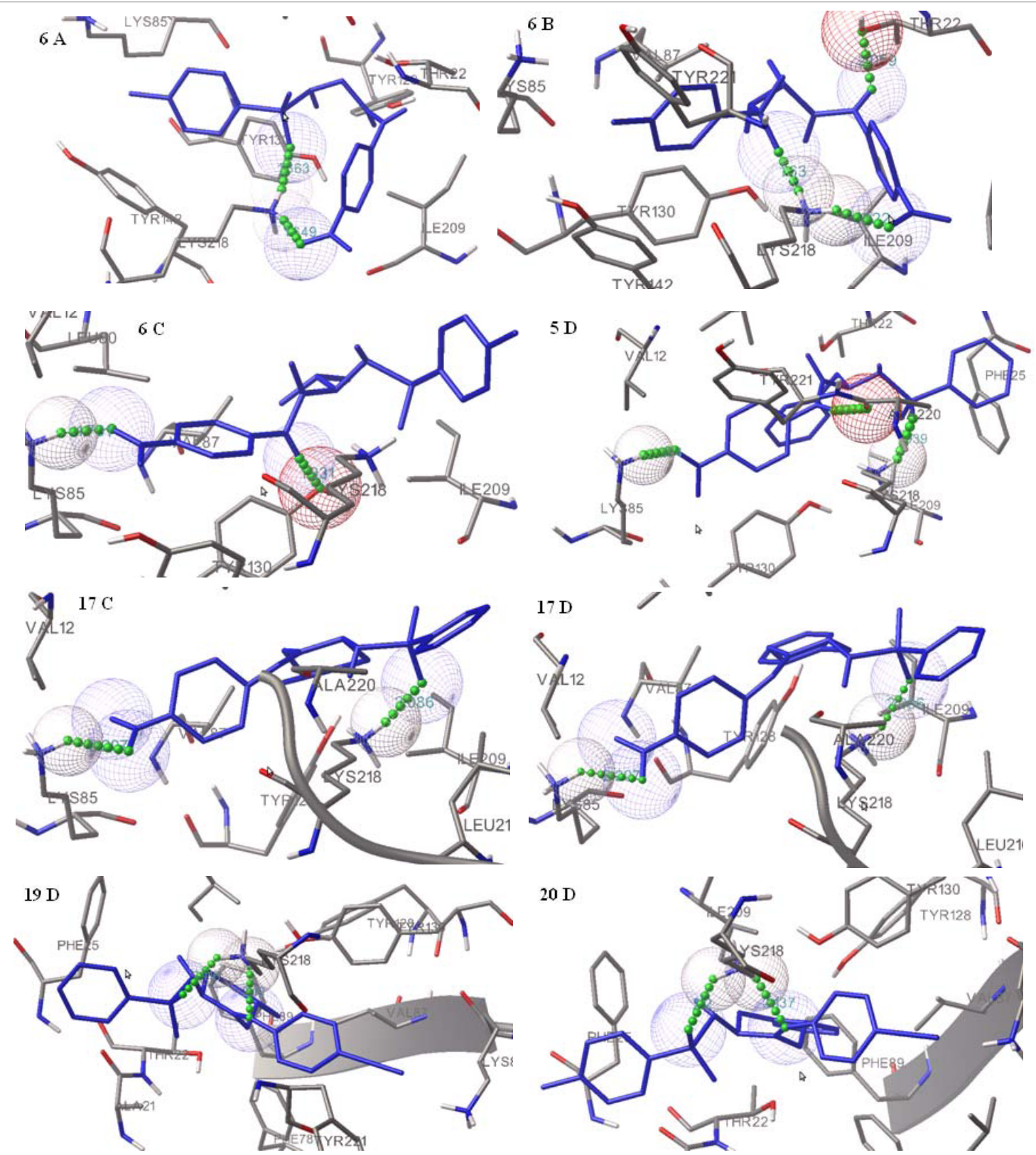

Figure 3: H-bond interactions between the amino acid residues at the binding pocket of juvenile hormone binding protein of $\mathrm{G}$. mellonella and best screened analogues of series $A$ to $D$ (juvenile hormone analogues are shown in blue. Hydrogen bond interactions between amino acid residue and ligands are shown in green-coloured arrow. 
Citation: Pamita A, Priyanka S (2013) Designing and Binding Mode Prediction of Juvenile Hormone Analogues as Potential Inhibitor for Galleria mellonella. J Comput Sci Syst Biol 6: 106-111. doi:10.4172/jcsb.1000107

However, analogues from 13-20 of all series, again shows uniform trend of lowering of binding energy profile. Greater lowering of energy has been observed for the proposed $\mathrm{C}$ and $\mathrm{D}$ series, as compared to $A$ and $B$ series. Overall it is the $R_{1}$ group which plays the important role for effective interactions inside the binding pocket. More is the interaction, greater will be the lowering in B.E. profile of the analogues, more stable will be the JHAs-JHBP complex. This stable complex will leads to the malfunction of the insect species, as it will hinder the metamorphosis process. This ultimately results in the control of insect pest species, i.e. G. mellonella.

Therefore, analogues 5 (D), 6 (C), 17 (C and D), 18 (D), 19 (D) and 20 (D) complexes having $-8.36 \mathrm{kcal} / \mathrm{mol},-8.22 \mathrm{kcal} / \mathrm{mol},-8.31 \mathrm{kcal} /$ $\mathrm{mol},-8.8 \mathrm{kcal} / \mathrm{mol},-8.7 \mathrm{kcal} / \mathrm{mol},-8.28 \mathrm{kcal} / \mathrm{mol},-8.55 \mathrm{kcal} / \mathrm{mol}$, respectively. This indicates that these seven analogues have a good binding free energy profile with JHBP of G. mellonella, these (JHAsJHBP) docking complexes are expected to be stable, and their binding modes are indicated to be reliable.

\section{Conclusion}

Nowadays, virtual screening is becoming one of the most powerful computational tools for the rapid discovery of novel and original chemical entities with potential activity. This technique is now used to understand the binding mode of active compounds and identify new hits. So far, application of a docking-based virtual screening approach, with the crystal structure of JHBP of G. mellonella has been published [16]. They have led chemists directly to consider those structural properties of a ligand that are essential for interacting with active site of target protein. This leads to the rationalization of the designing process by computer aided design. However, these approaches are limited by the accuracy with which the affinity of proposed ligands can be estimated. Since correct ranking of putative ligands for synthesis is a prerequisite for molecule design, there is a clear need for an objective method that is able to predict the binding affinity of a protein-ligand complex. We here proposed two series (C and D) of juvenile hormone analogues with different structural modification, keeping in view the nature of the lining of the binding pocket. These two series exhibit a good binding behavior over the synthetic IGRs. Based upon these results, our compounds are expected to exhibit better chemical control over the G. mellonella. These theoretical findings further need the biological support which will be reported subsequently.

\section{Acknowledgment}

Authors are highly thankful to the Director, N.I.T. Hamirpur for providing the necessary facilities to carry out the research work reported in the manuscript.

\section{References}

1. Ozyhar A, Kochman M (1987) Juvenile-hormone-binding protein from the hemolymph of Galleria mellonella (L) Isolation and characterization. Eur J Biochem 162: 675-682.

2. Wieczorek E, Kochman M (1991) Conformational change of the haemolymph juvenile-hormone-binding protein from Galleria mellonella (L). Eur J Biochem 201: 347-353.

3. Krzyzanowska D, Lisowski M, Kochman M (1998) UV-difference and CD spectroscopy studies on juvenile hormone binding to its carrier protein. J Pept Res 51: 96-102.
4. Kołodziejczyk R, Kochman M, Bujacz G, Dobryszycki P, Ozyhar A, et al. (2003) Crystallization and preliminary crystallographic studies of juvenile hormonebinding protein from Galleria mellonella haemolymph. Acta Crystallogr Sect D Biol Crystallogr 59: 519-521.

5. Kolodziejczyk R, Bujacz G, Jakob M, Ozyhar A, Jaskolski M, et al. (2008) Insect Juvenile hormone binding protein shows ancestral fold present in human lipidbinding proteins. J Mol Biol 377: 870-881.

6. Mahajan RK, Patial VP, Sharma P (2002) Juvenile hormone like substances: Part XV-Synthesis and biological activities of some juvenile hormone analogues containing sulfonamide feature. Indian J Chem 41B: 2635- 2641.

7. Awasthi P, Mahajan RK (2008) Synthesis of some sulfonamide insect juvenile hormone-Part I. Indian Journal of Chemistry 47B: 1291-1297.

8. Morris GM, Goodsell DS, Halliday RS, Huey R, Hart WE, et al. (1998) Automated docking using a lamarckian genetic algorithm and empirical binding free energy function. J Comput Chem 19: 1639-1662.

9. Carlos FL, Caballero J, Gonzalez-Nilo FD, Pessoa-Mahana CD, Perez-Acle T (2008) Docking and quantitative structure-activity relationship studies for the bisphenylbenzimidazole family of non-nucleoside inhibitors of HIV-1 reverse transcriptase. Chemical Biology and Drug Design 72: 360-369.

10. Sasorith S, Billas IM, Iwema T, Moras D, Wurtz JM (2002) Structure-based analysis of the ultraspiracle protein and docking studies of putative ligands. J Insect Sci 25: 1-11.

11. Garriga M, Caballero J (2011) Insights into the structure of urea-like compounds as inhibitors of the juvenile hormone epoxide hydrolase $(\mathrm{JHEH})$ of the tobacco hornworm Manduca sexta: Analysis of the binding modes and structureactivity relationships of the inhibitors by docking and CoMFA calculations. Chemosphere 82: 1604-1613.

12. Jenwitheesuk E, Samudrala R (2003) Improved prediction of HIV-I proteaseinhibitor binding energies by molecular dynamics simulations. BMC Struct Biol 3: 2 .

13. Hirano M, Hatakoshi M, Kawada H (1998) Pyriproxyfen and other juvenile hormone analogues. Review in Toxicology 2: 357-394.

14. De Clercq E (2001) New developments in anti-HIV chemotherapy. Curr Med Chem 8: 1543-1572.

15. Genc Y, Ozkanca R, Bekdemir Y (2008) Anti-microbial activity of some sulfonamide derivatives on clinical isolates of Staphylococus aureus. Ann Clin Microbiol Antimicrob 7: 17-22.

16. Awasthi $P$, Sharma $P$ (2012) In silico screening of the juvenile hormone analogues with juvenile hormone binding protein of Galleria mellonella-A docking study. SAR QSAR Environ Res 23: 607-625. 